\title{
Analysis of Electromagnetic Scattering Characteristics of Moving Target Group Based on Quasi-static Principle

\author{
Chaoyang Li
}

School of Computer Engineering and Science, Shanghai University, Shanghai 200444, China

rachy1989@163.com

\begin{abstract}
Keywords: Quasi-static principle; Moving target group; Multilevel fast multipole; Electromagnetic scattering; RCS
\end{abstract}

\begin{abstract}
The analysis of electromagnetic scattering characteristics of the static target after years of development has formed a mature and complete system, the electromagnetic scattering characteristics of target motion analysis in the last few years has become a hot and difficult problem for researchers and engineers. This paper presents an analysis method of quasi static principle of moving target group of electromagnetic scattering characteristics based on the multilevel fast multipole algorithm to accelerate the computation of RCS characteristics at different time of low velocity moving target group, and then analyzes different motion state of the target group of the electromagnetic scattering effect. Numerical experiment results show the effectiveness of the proposed method. Is not to RCS with the motion of a moving target group; non identical to RCS with the motion of a moving target in different groups will have the corresponding migration, and in the same incident wave and observation angle under the condition of different migration effect of different state of motion generated.
\end{abstract}

\section{Chapter 1 Introduction}

The analysis of target electromagnetic scattering characteristics is of great significance to both scientific research and engineering application. In the aerospace, marine ships, ground transportation, military weapons and other practical applications, the analysis of the target electromagnetic scattering characteristics is particularly important. This not only helps to enhance the design of high-performance radar systems, but the more accurate the detection and identification of targets, the more important the improvement of people's lives and property, which is evident in the aerospace and military fields.

Traditional measurement methods, although the most authentic, but often will spend a great deal of human, financial and material resources, and the complexity of the real situation will lead to difficult to accurately reproduce the experimental results. What is more, some measurements can not be measured, such as some highly dangerous or costly measurements, due to objective difficulties, and the measurement conditions are not allowed. This stimulates the birth of numerical simulation experiments. Numerical simulation to low-cost, high-precision and reproducible advantages all walks of life researchers and engineers of all ages.

The numerical simulation of the electromagnetic scattering characteristics of static targets has developed a set of mature and complete theoretical systems after years of development. The traditional static target electromagnetic scattering characteristic analysis is fixed in the relative position of the target and the radar, the relative position of the target and the target is fixed, and the target itself remains stationary, there is no translation, rotation, rolling, twisting, vibration and motion characteristics. As long as the incident angle of incidence of electromagnetic waves, polarization direction and frequency, you can choose the appropriate method to accurately calculate the target in the corresponding time or frequency domain scattering, can truly measure the value of high precision fitting is very popular in the industry.

However, in reality, it is often necessary to analyze the electromagnetic scattering characteristics of the target under moving conditions, such as aircraft flying in the sky, ships navigating on the sea surface, and missiles tracking targets. We can not stop these targets to measure the electromagnetic scattering characteristics of them, nor directly with the traditional static target of electromagnetic scattering analysis method to study, because the movement of the electromagnetic scattering 
characteristics of a great impact. For the purpose of engineering requirements, more and more researchers and engineers are engaged in the study of electromagnetic scattering characteristics of moving objects.

The research focus of common electromagnetic scattering of moving objects are incident wave frequency, target moving velocity, target fretting and attitude angle change. The common approach is to create two coordinate systems: the radar coordinate system and the target coordinate system. The motion state of the target is expressed by the position and angle relationship of two coordinate systems. The first step is to convert the incident electromagnetic wave of the radar coordinate system into the incident electromagnetic wave of the target coordinate system, and then calculate the induced current under the target system. And then the radar cross section RCS is calculated. The differences of dynamic and static RCS characteristics in undulating target detection performance evaluation are analyzed emphatically[1]. The high frequency approximation method is used to calculate the electromagnetic scattering of moving objects[2-3]. The quasi-static principle and the fast multipole algorithm are used to calculate the electromagnetic scattering characteristics of moving objects of arbitrary shape[4-7]. According to the statistic distribution of static and dynamic RCS difference, the method of calculating confidence interval and mathematical expectation value is put forward[8]. Throughout the domestic and foreign scholars in the field of research on the status quo of moving objects, mostly focusing on a single moving target of the electromagnetic scattering of accurate solutions, while the moving target group of electromagnetic scattering characteristics of little research.

For this reason, the method proposed in this paper is to apply the quasi-static principle to the moving target group, to discretize the moving process of the target group into static state at several different moments, and combine the fast multipole algorithm to solve the And then the effect of different motion states on the EM scattering characteristics of the moving target group is analyzed.

\section{Chapter 2 Quasi-static principle}

The speed of movement of macroscopic objects common in everyday life is negligible relative to the speed of electromagnetic waves (the speed of light). When a constant frequency of electromagnetic radiation in the moving object surface, it will stimulate the surface of the induced current, can be considered at a time the induced current is not subject to the moment the speed of movement of objects and instantly stabilized, and the speed of time Will not cause great errors. So that the electromagnetic scattering characteristic of the moving object can be approximately equivalent to the static electromagnetic scattering characteristic of the moving object in some series of observation time slices. This is the basic idea of quasi-static principle. [4-7]

According to the mutual relationship between the radar and the target coordinate system, it can theoretically solve the electromagnetic scattering problem of any dynamic target accurately. Quasi-static principle to a certain extent, simplifies the process. The rationality of using the quasi-static principle to solve the electromagnetic scattering of moving objects is discussed in detail[7].

For a moving target, the change in distance from the half-wavelength of the radar will result in a $360^{\circ}$ phase change. If the change in distance from the target due to motion is much smaller than the radar operating wavelength for a short period of time considered, the time-domain solution is simulated in a series of stationary positions in discrete-time steps. In order to ensure the high resolution time-frequency distribution do not overlap, at least 8 points in a wavelength, to ensure that the high resolution time-frequency distribution does not overlap. For the actual target, the moving speed of the target surface relative to the radar is more complex than that of the point target, and the target attitude change will produce complicated amplitude and polarization modulation to the incident electromagnetic wave, so the actual moving target will cause the micro-Doppler spectral broadening. In order to distinguish the fine target of the actual characteristics of the fine, the sampling rate should be higher than the point target case of the sampling rate[7]. 


\section{Chapter 3 Multilevel fast multipole algorithm}

As we all know, when we do any numerical simulation, because of the particularity of the computer, we have to convert the continuous practical problems into discrete approximation problems so that the computer can deal with it. This is why we need to discretize the integral equation or the differential equation when we compute the electromagnetic scattering of the target. For different equations because of the different basis functions used, simulation targets often have different ways of segmentation. The common methods of mesh generation are triangular mesh generation, parameter two mesh generations, tetrahedral mesh generation and so on. For example, the surface integral equation using the RWG basis function is to divide the continuous target surface into triangular mesh elements, and one side of the mesh represents an unknown quantity.

Assuming there are $\mathrm{N}$ unknowns in the scattering problem of the target, the problem is solved directly by the Moment of Method (MOM)[9], which is equivalent to solving the problem of N-body mutual-coupling, the resulting impedance matrix is $N \times N$, the storage complexity is $O\left(N^{2}\right)$, and since the impedance matrix is dense, the time complexity of the direct method (Gaussian elimination or LU decomposition) is $O\left(N^{3}\right)$. Although the exact solution of the problem can be obtained by using the method of moments, such a large amount of storage and computation can greatly limit the scale of the problem. Even with the rapid development of computer technology, memory and computing power are greatly improved. However, the problem that a PC can solve by using the method of moments can not exceed the scale of 100,000. Secondly, due to the nature of the N-matrix method and the density of the resulting matrix, it is also in the field of parallel computing is stagnant. However, in the face of strong demand for scientific research and engineering applications, computational electromagnetism is urgently needed to find a new and efficient algorithm to replace the method of moments.

So the fast multipole algorithm (FMA), one of the Top 10 algorithms in the twentieth century, came into being[10]. The fast multipole algorithm is based on the method of moments, and the interaction of all scatters is divided into near field and far field. In general, fast multipole algorithm is to target the body with a suitable size of the cube cover up, and then the cube is divided into side length $0.5 \lambda$ (for the simulation of the incident wavelength) around the cube. The scatters are assigned to the corresponding small cubes based on the position of the center of the sub scatter (if the RWG basis function is used, that is, the midpoint of each side of the triangle mesh) to find a non-empty cube, called a group. Adjacent non-empty cube is called the adjacent group, non-adjacent non-empty cube is called far-field group. When the sub scatter $\mathrm{i}$ is calculated for the sub scatter $i$, the positional relationship between the self-scatter $j$ and the sub-scatter $i$ is calculated by using the method of moments directly if it belongs to the neighboring group. If the group belongs to the far-field group, the additive theorem first gathers the action of the sub-scatter $\mathrm{j}$ into the center of its group, and then transfers the amount of polymerization to the group center of the sub-scatter $\mathrm{i}$, and finally the transfer amount obtained from the group center of the sub-scatter $\mathrm{i}$ to the sub-scatter $\mathrm{i}$. As a result, this "switch" mechanism can be the method of moments all directly scatter between conversion into the indirect effects of fast multipole algorithm, this approach not only speed up the calculation of $\mathrm{N}$ body of lotus root, and aggregation, transfer, allocation of the three process are sparse matrix, greatly to reduce the amount of storage, also facilitate the use of iterative method for solving equations. It turns out that this single-layer fast multipole algorithm reduces the storage complexity from $O\left(N^{2}\right)$ to $O\left(N^{1.5}\right)$ and reduces the time complexity from $O\left(N^{3}\right)$ to $O\left(N^{1.5}\right)$ for the method of moments. Coupled with the fast multipole is a sparse matrix, iterative method can be easily solved using parallel computing, which makes the limited computer resources to solve the problem size has been greatly improved.

However, the single-layer fast multipole algorithm does not take advantage of this "switch" mechanism to its fullest. As a result, Professor Weng Cho Chew and other scholars have proposed an improved version of the multilevel fast multipole algorithm (MLFMA) based on the single-level fast multipole algorithm [11]. As the name suggests, multilevel fast multipole algorithm is a fast multipole sub-algorithm to achieve. In the case of a 3D object (similar to a two-dimensional object), 
a multilevel fast multipole algorithm is designed by first enclosing the target with a properly sized cube, denoting the layer 0 , and then dividing the cube into eight sub- Cube, recorded as the first layer; then the first layer of each cube is divided into smaller 8 small cube, recorded as the second layer ....... and so on, until the first n-layer c-side length is about $0.5 \lambda$ stop Segmentation. Each layer of the cube is divided into three positional relationships. This layer directly adjacent to the two layer cube each other for the "adjacent group", adjacent to the parent layer but this layer is not adjacent to the two cube mutual "distant group", the parent layer is not adjacent to this layer is not the two adjacent cubes are "other groups". Like the fast multipole algorithm, the multilevel fast multipole algorithm also decomposes the matrix and vector multiplication into three processes: aggregation, transfer and configuration. However, the aggregation process in the most delicate layer, and then complete the father through the interpolation layer aggregation, aggregation process to the second layer stop.The transfer process is exactly the same as that of the fast multipole algorithm, except that the multilevel fast multipole algorithm is only carried out between the distant relatives of the same layer. The configuration process is opposite to the aggregation process, starting from the second layer, through the inverse interpolation layer down to the next layer configuration, until the n-layer. Multilevel fast multipole algorithm will be "multilevel switch" mechanism to the extreme advantage, making the storage complexity of the algorithm reduced to $O(N)$, time complexity reduced to $O(N \log N)$. These remarkable advantages, coupled with the high accuracy of the multilevel fast multipole algorithm, are particularly suitable for solving large-scale and even large-scale unknowns, whether acoustic, electromagnetic, or any other N-body interconnection problems. With the multilevel fast multipole algorithm, it is easy to solve the problem of millions of orders on a PC, and the parallel implementation of the algorithm, such as MPI, OpenMP and GPU, or combinations thereof, solves the billion- or billion- Scale super-large-scale problems become possible.

\section{Chapter 4 Numerical Simulation: Electromagnetic Scattering Analysis of Moving Two - Ball Target Group}

According to the quasi-static principle, the velocity of the moving target group is negligible relative to the speed of the electromagnetic wave (the speed of light). It is considered that the low-speed moving target group is stationary at a certain moment and the electromagnetic scattering of the stationary target group at that moment can be approximated Represents the electromagnetic scattering of the moving target group at that time. In this way, we can give the motion equations of moving objects in the moving target group, and then according to the above sampling rate requirements, each wavelength sampling rate is greater than 8 . In this paper, in order to simplify the simulation, we assume that the target in the experiment is a rigid body, and do not consider the change of the attitude angle in the motion, that is, all the movements are translational.

Double ball target group, radius is $r_{1}=r_{2}=0.5 \mathrm{~m}$, the initial state of the center were $O_{1}(-1,0,0)$, $\mathrm{O}_{2}(1,0,0)$, as shown in Fig.1. Simulation of the incident wave frequency of $f=300 \mathrm{MHz}$, the incidence angle of $\theta^{i}=0^{\circ}, \phi=90^{\circ}$ (here said $\theta$ and $Z$ axis is the angle between the half axis, $\phi$ expression and $x$ axis positive half axis angle, $\left(0^{\circ}, 90^{\circ}\right)$ said inverse $z$ axis direction of incidence). Observation angle range $0^{\circ} \leq \theta \leq 180^{\circ}, 90^{\circ} \leq \phi \leq 90^{\circ}$. Due to the incident wavelength of $\lambda=1 \mathrm{~m}$, we take 10 sampling points per wavelength, that is, the sampling interval $\Delta=0.1 \mathrm{~m}$. 


\section{double sphere}

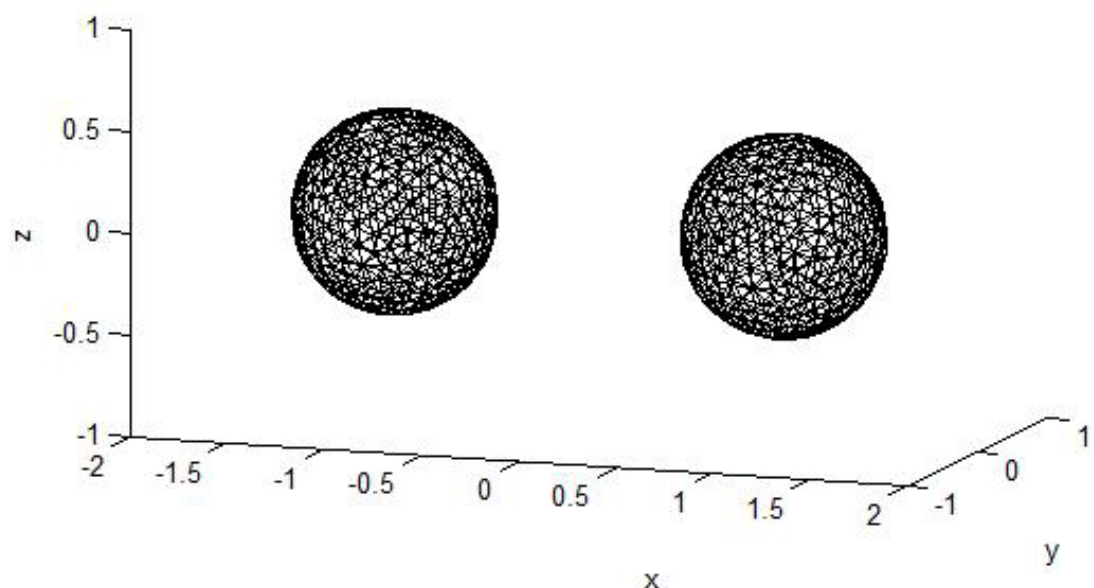

Fig.1 double sphere system
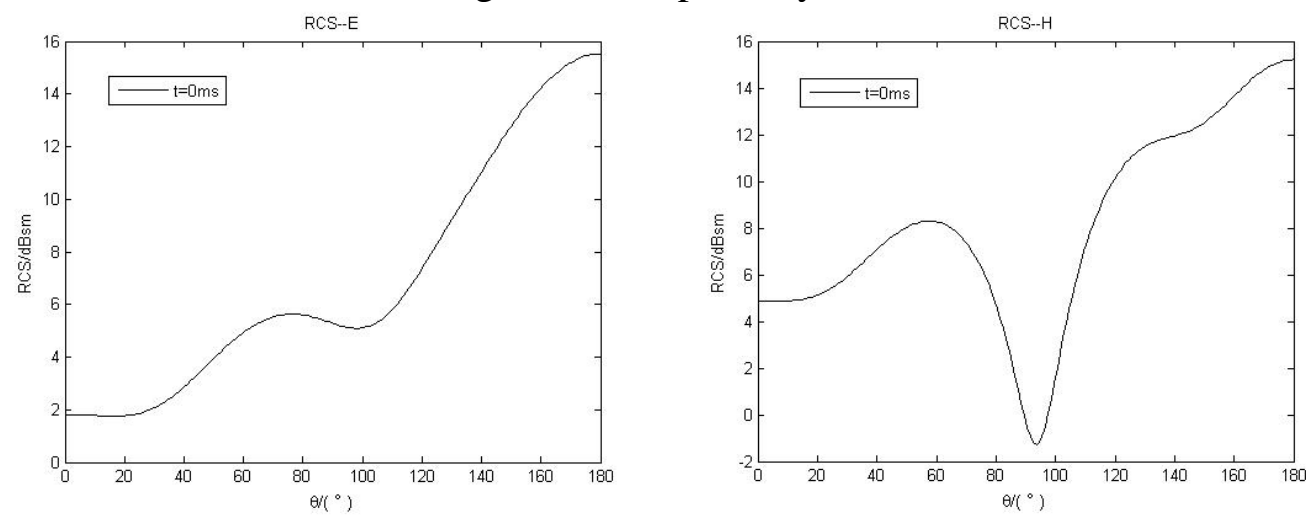

Fig.2 RCS of double sphere at time $\mathrm{t}=0 \mathrm{~ms}$

4.1 Same direction movement

4.1.1 Assuming the double ball system double_sphere forward motion along the $\mathrm{X}$ axis, the velocity is $v_{x}=+100 \mathrm{~m} / \mathrm{s}$, the motion trajectory equation is

$$
\left\{\begin{array}{l}
x(t)=x(0)+v_{x} t \\
y(t)=y(0) \\
z(t)=z(0)
\end{array}\right.
$$

Then in order to guarantee the sampling interval $\Delta=0.1 \mathrm{~m}$, need to meet $\Delta=v_{x} \cdot \Delta t$, so the sampling time interval $\Delta t=1 \mathrm{~ms}$.
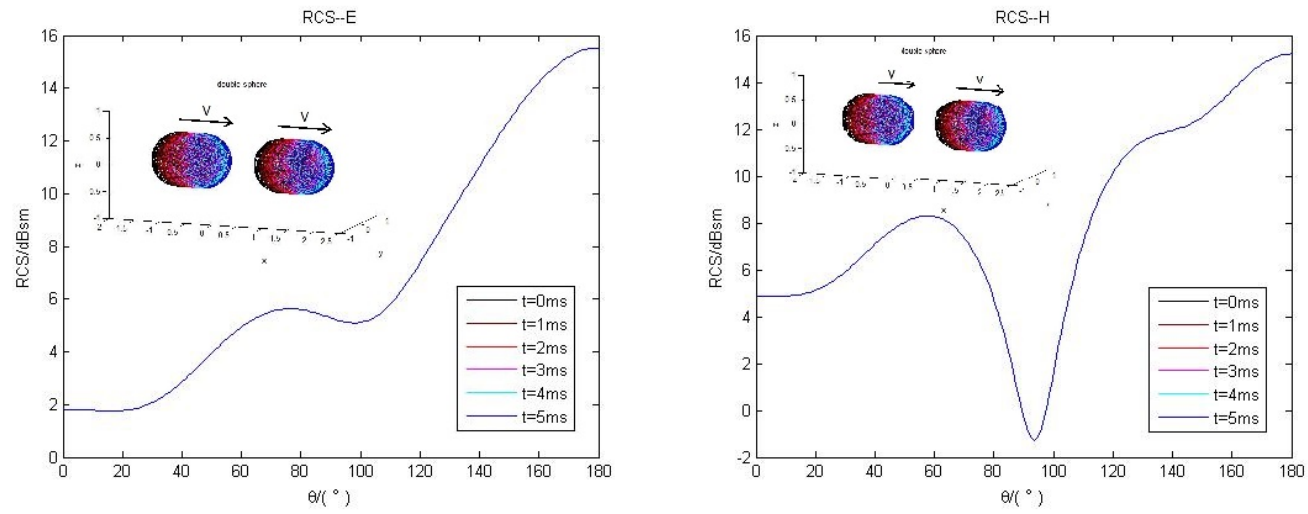

Fig.3 RCS of double sphere moving along $+x$ axis

Analysis: the electromagnetic scattering characteristics of the double-sphere system moving in the same direction along the $\mathrm{x}$-axis are constant.

4.1.2 Assuming the double ball system double_sphere forward motion along the Y axis, the velocity 
is $v_{y}=+100 \mathrm{~m} / \mathrm{s}$, the motion trajectory equation is

$$
\left\{\begin{array}{l}
x(t)=x(0) \\
y(t)=y(0)+v_{y} t \\
z(t)=z(0)
\end{array}\right.
$$

Then in order to guarantee the sampling interval $\Delta=0.1 \mathrm{~m}$, need to meet $\Delta=v_{y} \cdot \Delta t$, so the sampling time interval $\Delta t=1 \mathrm{~ms}$.
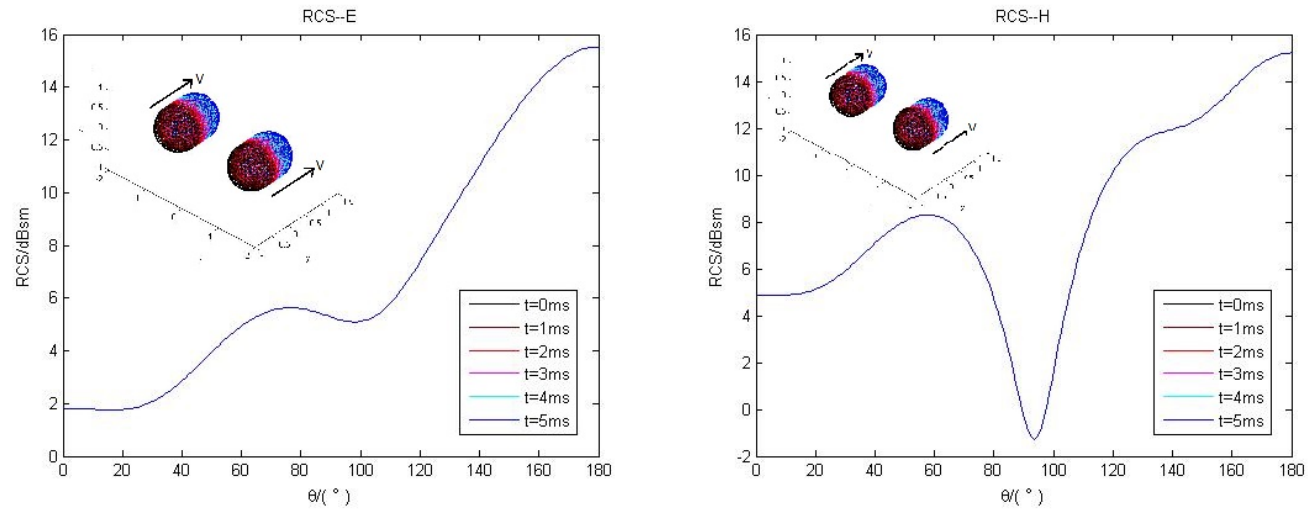

Fig.4 RCS of double sphere moving along +y axis

Analysis: the electromagnetic scattering characteristics of the double-sphere system moving in the same direction along the $y$-axis are constant.

4.1.3 Assuming the double ball system double_sphere forward motion along the $\mathrm{Z}$ axis, the velocity is $v_{z}=+100 \mathrm{~m} / \mathrm{s}$, the motion trajectory equation is

$$
\left\{\begin{array}{l}
x(t)=x(0) \\
y(t)=y(0) \\
z(t)=z(0)+v_{z} t
\end{array}\right.
$$

Then in order to guarantee the sampling interval $\Delta=0.1 \mathrm{~m}$, need to meet $\Delta=v_{z} \cdot \Delta t$, so the sampling time interval $\Delta t=1 \mathrm{~ms}$.
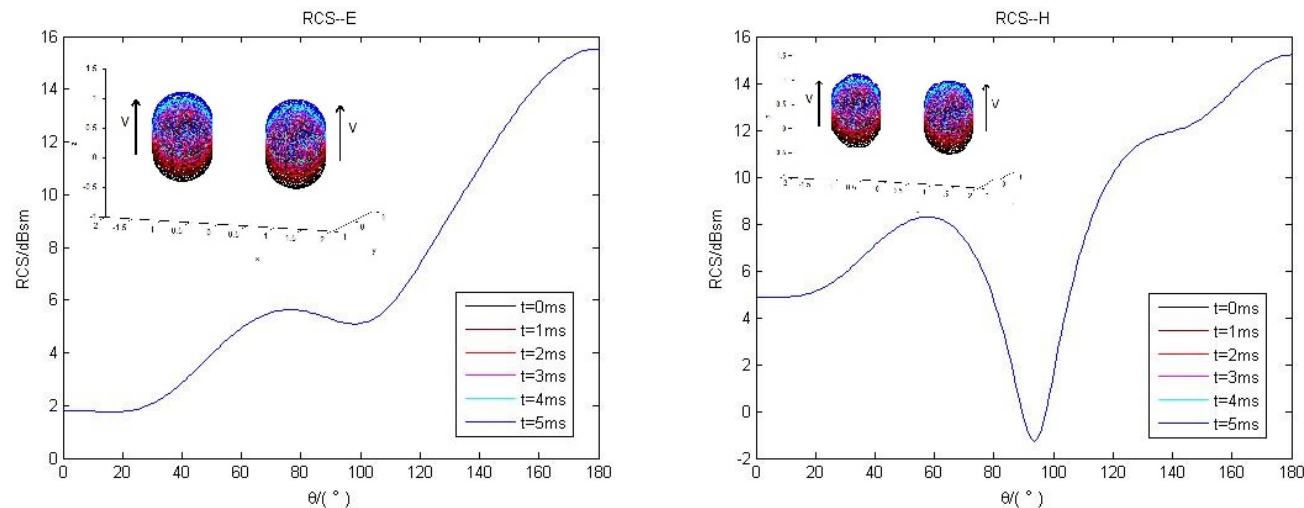

Fig.5 RCS of double sphere moving along $+\mathrm{z}$ axis

Analysis: the electromagnetic scattering characteristics of the double-sphere system moving in the same direction along the z-axis are constant.

4.2 Reverse motion

4.2.1 Assuming the double ball system double_sphere the reverse motion along the $\mathrm{X}$ axis, the velocity is $v_{x}= \pm 100 \mathrm{~m} / \mathrm{s}$, the motion trajectory equation is

$$
\left\{\begin{array}{l}
x(t)=x(0)+v_{x} t \\
y(t)=y(0) \\
z(t)=z(0)
\end{array}\right.
$$


Then in order to guarantee the sampling interval $\Delta=0.1 \mathrm{~m}$, need to meet $\Delta=v_{x} \cdot \Delta t$, so the sampling time interval $\Delta t=1 \mathrm{~ms}$.
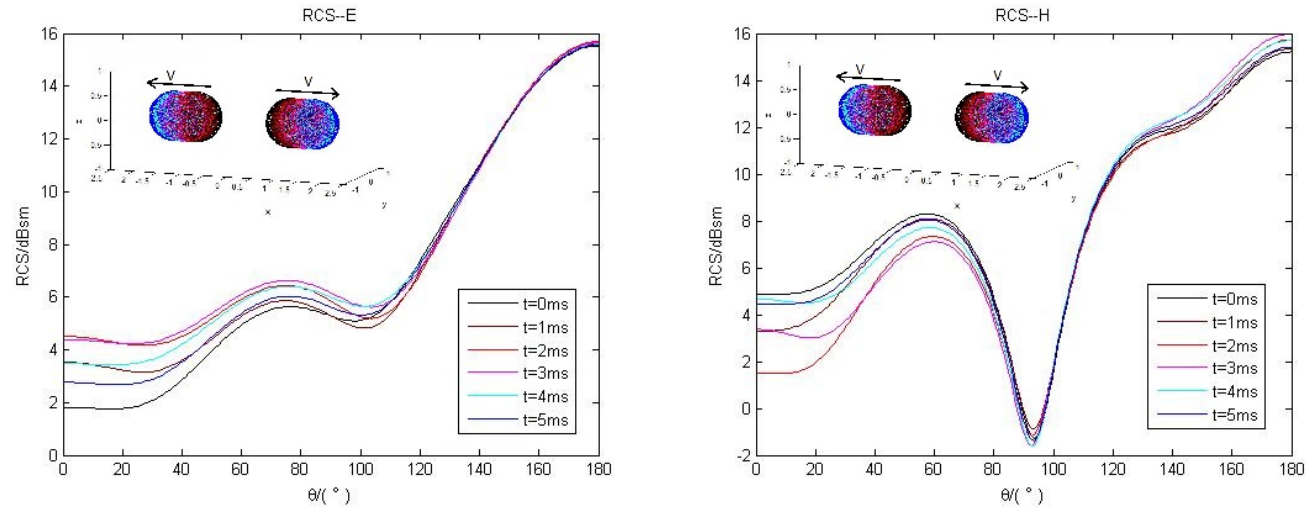

Fig.6 RCS of double sphere moving along $-\mathrm{x}$ and $+\mathrm{x}$ axis

Analysis: A double-sphere system moving in the opposite direction along the $x$-axis. The direction of movement (x-axis) is perpendicular to the observation plane $\left(0^{\circ} \leq \theta \leq 180^{\circ}\right.$, $90^{\circ} \leq \phi \leq 90^{\circ}$ is the yoz plane). RCS has a corresponding small amplitude migration.

4.2.2 Assuming the double ball system double_sphere the reverse motion along the $\mathrm{Y}$ axis, the velocity is $v_{y}= \pm 100 \mathrm{~m} / \mathrm{s}$, the motion trajectory equation is

$$
\left\{\begin{array}{l}
x(t)=x(0) \\
y(t)=y(0)+v_{y} t \\
z(t)=z(0)
\end{array}\right.
$$

Then in order to guarantee the sampling interval $\Delta=0.1 \mathrm{~m}$, need to meet $\Delta=v_{y} \cdot \Delta t$, so the sampling time interval $\Delta t=1 \mathrm{~ms}$.
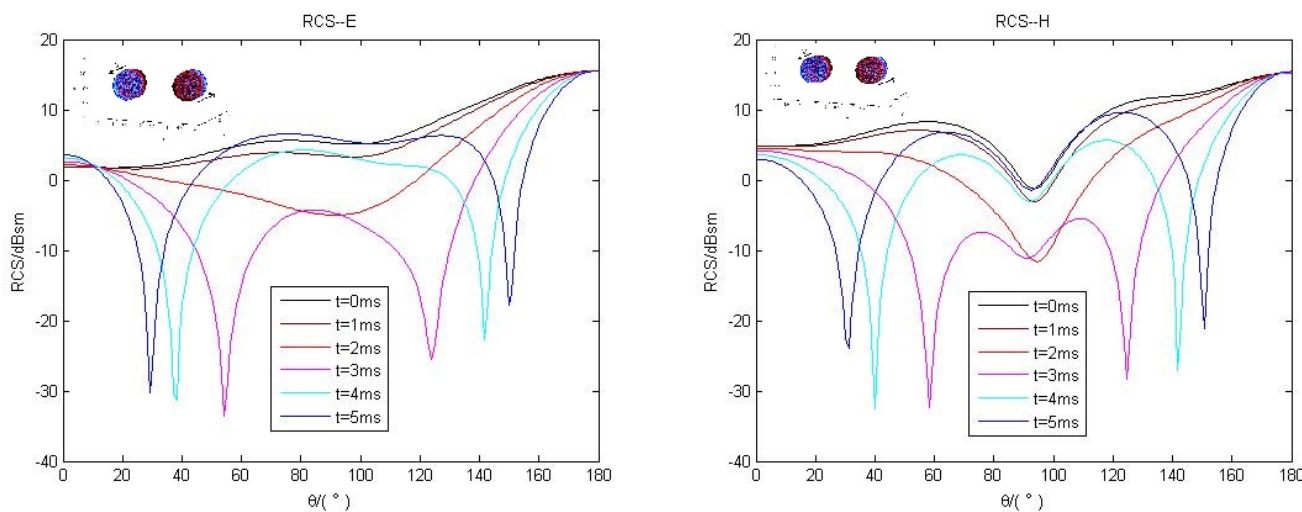

Fig.7 RCS of double sphere moving along $-\mathrm{y}$ and $+\mathrm{y}$ axis

Analysis: In the double-sphere system moving in the opposite direction along the y-axis, the movement direction (y-axis) in the observation plane $\left(0^{\circ} \leq \theta \leq 180^{\circ}, 90^{\circ} \leq \phi \leq 90^{\circ}\right.$ represents the yoz plane). And $t \geq 3 m s$, the relative position of the two ball changes greater than $0.5 \lambda$, RCS will become no longer credible.

4.2.3 Assuming the double ball system double_sphere the reverse motion along the $\mathrm{Z}$ axis, the velocity is $v_{z}= \pm 100 \mathrm{~m} / \mathrm{s}$, the motion trajectory equation is

$$
\left\{\begin{array}{l}
x(t)=x(0) \\
y(t)=y(0) \\
z(t)=z(0)+v_{z} t
\end{array}\right.
$$

Then in order to guarantee the sampling interval $\Delta=0.1 \mathrm{~m}$, need to meet $\Delta=v_{z} \cdot \Delta t$, so the sampling time interval $\Delta t=1 \mathrm{~ms}$. 

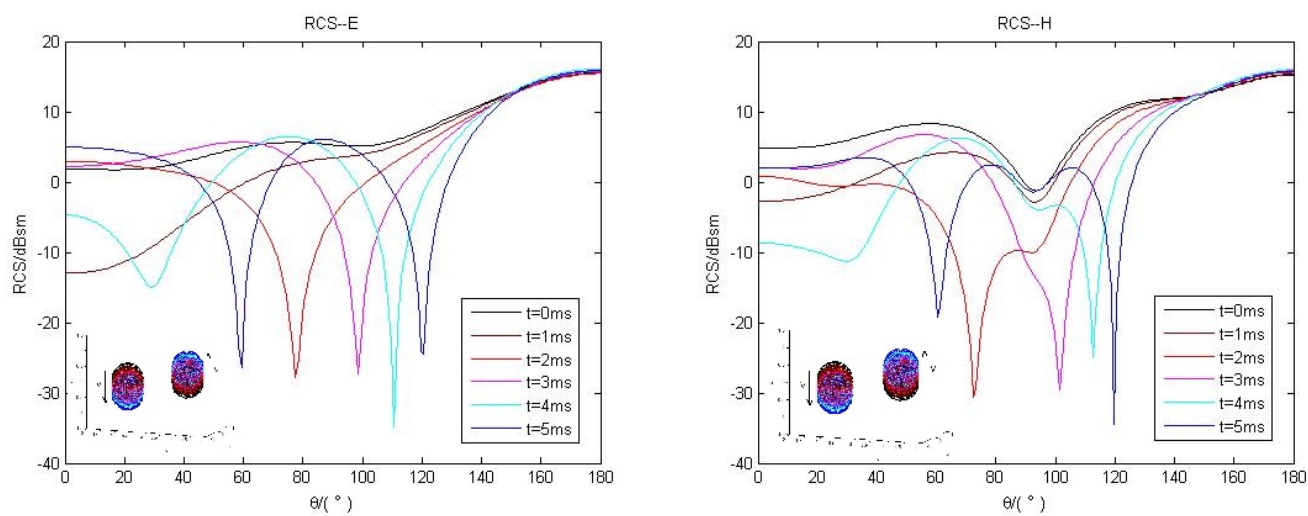

Fig.8 RCS of double sphere moving along $-\mathrm{z}$ and $+\mathrm{z}$ axis

Analysis: In the double-sphere system moving in the opposite direction along the z-axis, the movement direction (z-axis) in the observation plane $\left(0^{\circ} \leq \theta \leq 180^{\circ}, 90^{\circ} \leq \phi \leq 90^{\circ}\right.$ represents the yoz plane). And $t \geq 3 \mathrm{~ms}$, the relative position of the two ball changes greater than $0.5 \lambda$, RCS will become no longer credible.

\subsection{Arbitrary motion}

4.3.1 Assuming that the double ball system double_sphere in the xoy plane, respectively, along the xoy first quadrant and the second quadrant of the diagonal direction, the speed of $v=+100 \mathrm{~m} / \mathrm{s}$, the motion trajectory equation

$$
\left\{\begin{array}{l}
x(t)=x(0)+v_{x} t \\
y(t)=y(0)+v_{y} t \\
z(t)=z(0)
\end{array}\right.
$$

Then in order to guarantee the sampling interval $\Delta=0.1 \mathrm{~m}$, need to meet $\Delta=v \cdot \Delta t$, so the sampling time interval $\Delta t=1 \mathrm{~ms}$.
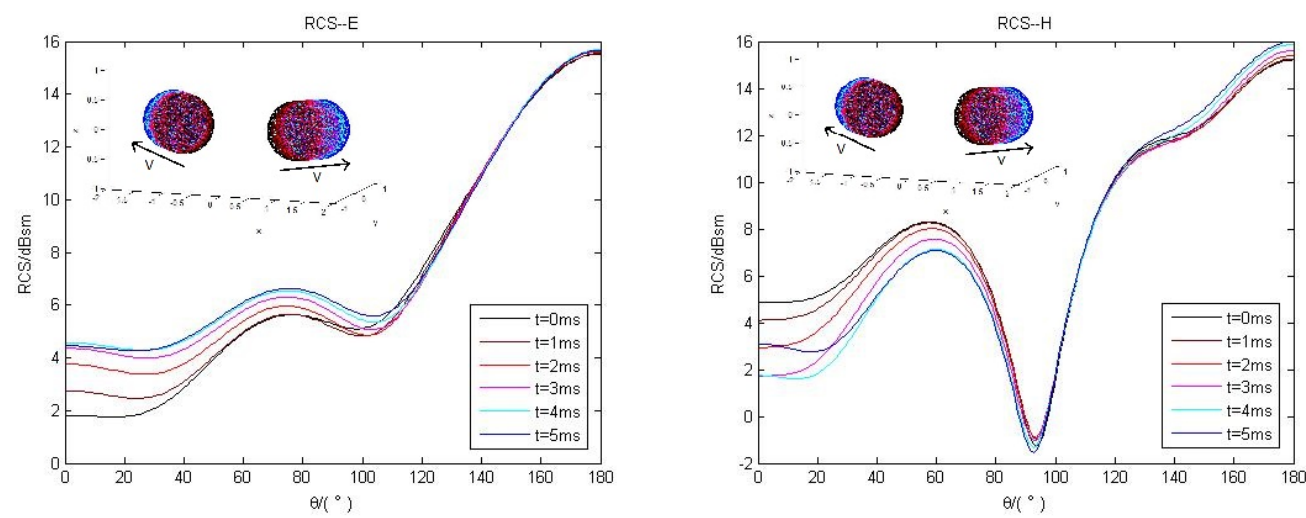

Fig.9 RCS of double sphere moving by diagonals of plane xoy

Analysis: the RCS of the two-sphere system with the diagonal movement of the first and second quadrants along the xoy plane is correspondingly migrated.

4.3.2 Assuming that the double ball system double_sphere is in the o-xyz Descartes coordinate system along the diagonal direction of the first and the second limit respectively, the velocity is $v=+100 \mathrm{~m} / \mathrm{s}$, and the motion trajectory equation is

$$
\left\{\begin{array}{l}
x(t)=x(0)+v_{x} t \\
y(t)=y(0)+v_{y} t \\
z(t)=z(0)+v_{z} t
\end{array}\right.
$$

Then in order to guarantee the sampling interval $\Delta=0.1 \mathrm{~m}$, need to meet $\Delta=v \cdot \Delta t$, so the sampling time interval $\Delta t=1 \mathrm{~ms}$. 

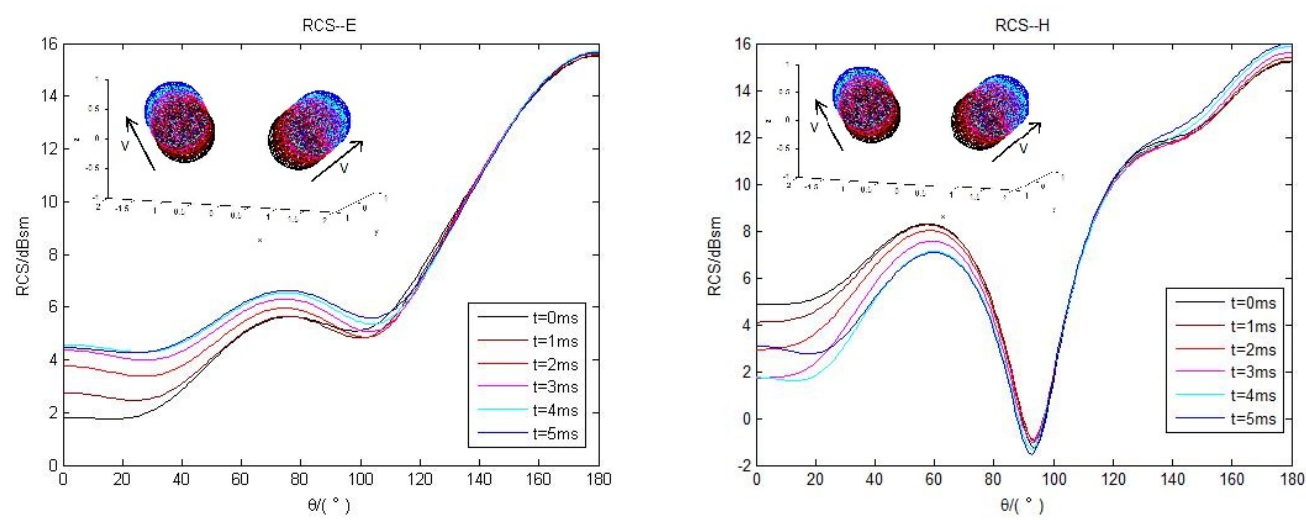

Fig.10 RCS of double sphere moving by diagonals of Cartesian xyz

Analysis: The RCS of the two-ball system along the first and second trapezoidal motion along the Cartesian coordinate system xyz has a corresponding migration.

\section{Chapter 5 Conclusion}

The analysis of electromagnetic scattering characteristics of moving targets is complex and has always been the research hotspots and difficulties of researchers and engineers. Unlike the electromagnetic scattering analysis of static targets, the electromagnetic scattering characteristics of moving objects are closely related to the motion state of the target itself. The change of the distance of the moving target about $0.5 \lambda$ from the radar will cause the RCS to change over and over again. Quasi-static principle combined with fast multipole method is the best choice to solve the electromagnetic scattering characteristics of moving objects. Based on previous studies, this paper presents an approximate method to solve the electromagnetic scattering characteristics of a moving target group using the quasi-static principle and fast multipole method. The effectiveness of the method is validated by numerical experiments.

The results of numerical experiments show that the RCS of the target group is independent of its motion state, while the target of the non-co-moving target group is relatively stationary. When the incident wave is invariant and the observation angle is constant, The RCS of the target group will migrate with the movement state of the target group, and the velocity of the target is parallel to the component of the observation plane than the component perpendicular to the observation plane. If the incident angle is constant, the impact of the RCS of the moving target group is greater.

More precise analysis of the electromagnetic scattering characteristics of the moving target group, need to consider the movement of the target group of fine, such as speed, movement direction, jogging characteristics and movement posture. In addition, the depth learning algorithm can be used to analyze the target group of electromagnetic scattering characteristics, and extract the movement characteristics, as the basis for forecasting the movement of the target group. Due to limited space, these elements will be given in the follow-up study.

\section{References}

[1] DAI Chong, XU Zhen-hai, XIAO Shun-ping. Analysis for Differences between Dynamic and Static RCS Characteristic of Radar Target. JOURNAL OF SIGNAL PROCESSING. Vol.29 (2013) No.9, p.1256-1263.

[2] C. Fang, X. Zhao, N. Xia, et al. Scattering of Electromagnetic Plane Waves by Moving Objects in High Frequency. 2008 China-Japan Joint Microwave Conference, Shanghai, 2008, p.298-300.

[3] BU Hong-mei, WANG Xiao-bin, LIANG Zi-chang. Analysis on Electromagnetic Scattering Characteristic of Spacecraft. AEROSPACE SHANGHAI. Vol.27 (2010) No.1,p26-30.

[4] XIANG Dao-pu, ZHOU Dong-ming, HE Jian-guo. The Application of MLFMA in Doppler Echo Simulation with Motion Body Target. JOURNAL OF NATIONAL UNIVERSITY OF DEFENSE TECHNOLOGY. Vol.32 (2010) No.2,p114-118. 
[5] XIANG Dao-pu, ZHOU Dong-ming, HE Jian-guo. Doppler Echo Simulation with Motion Body Target. JOURNAL OF MICROWAVES. Vol.26 (2010) No.3, p73-76.

[6] XIANG Dao-pu, ZHOU Dong-ming, HE Jian-guo. Application of MLFMA in Helicopter Doppler echo simulation. CHINESE JOURNAL OF RADIO SCIENCE. Vol.25 (2010) No.6,p1193-1198.

[7] ZHOU Cheng-hong, QIAN Wei-ping, GUO Yong-qiang. EM Scattering Model of Dynamic Rigid Target. Journal of Sichuan Ordnance. Vol.36 (2015) No.11, p138-140.

[8] LIU Jia, FANG Ning, XIE Yong-jun, et al. Dynamic target RCS characteristic analysis under the influence of attitude perturbation. Systems Engineering and Electronics. Vol.37 (2015) No.4, p775-781.

[9] SHENG Xin-qing. A Brief Treatise on Computational Electromagnetics (the second edition). Press of University of Science and Technology of China, 2008, p17-90.

[10] Rokhlin V. Rapid solution of integral equations of scattering theoryin two dimensions. Journal Computational Physics. Vol.86 (1990) No.2, p414-439.

[11] Song J M, Chew W C. Multilevel fast-multipole algorithm for solving combined field integral equations of electromagnetic scattering. Microwave Opt. Technol. Lett.,Vol.10 (1995) No.1, p14-19. 